\title{
EFEITOS DO TREINAMENTO PROPRIOCEPTIVO NA ESTABILIDADE DO TORNOZELO EM ATLETAS DE VOLEIBOL
}

EFFECTS OF PROPRIOCEPTIVE TRAINING ON THE STABILITY OF THE ANKLE IN

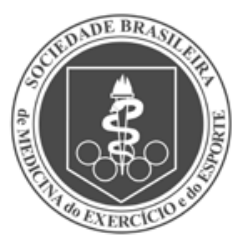

Artigo Original

\author{
VOLLEYBALL PLAYERS
}

\author{
EFECTOS DEL ENTRENAMIENTO PROPIOCEPTIVO SOBRE LA ESTABILIDAD DEL \\ TOBILLO EN JUGADORES DE VOLEIBOL
}

\author{
Mariana Michalski Peres \\ (Fisioterapeuta) \\ Lisiane Cecchini² $^{2}$ \\ (Fisioterapeuta) \\ Ivan Pacheco 2,3 \\ (Médico Ortopedista e \\ Traumatologista) \\ Adriana Moré Pacheco ${ }^{1}$ \\ (Fisioterapeuta)
}

1. Universidade Federal do Rio Grande do Sul, Porto Alegre,

RS, Brasil

2. Grêmio Náutico União, Porto

Alegre, RS, Brasil

3. Federação Gaúcha de Futebol,

Porto Alegre, RS, Brasil

\section{Correspondência:}

Adriana Moré Pacheco, Escola de Educação Física, Universidade Federal do Rio Grande do Sul, Rua Felizardo, 750. 90690-200 Porto

Alegre, RS, Brasil.

adrimpacheco@ufrgs.br

\section{RESUMO}

Introdução: Nos esportes onde há contato entre os atletas, como no voleibol, a entorse por mecanismo de inversão é a lesão mais frequente e o tornozelo é a articulação mais acometida quando comparada com as outras, acarretando déficits importantes como a instabilidade articular. Programas de treinamento proprioceptivo têm sido amplamente utilizados para minimizar essas questões. Objetivo: Avaliar o efeito de um treinamento proprioceptivo na estabilidade articular do tornozelo por meio do teste Star Excusion Balance Test (SEBT) em atletas de voleibol. Métodos: Foram avaliadas 11 atletas de voleibol de um clube poliesportivo da cidade de Porto Alegre, RS, Brasil, por meio de um teste de avaliação para estabilidade articular do tornozelo, SEBT. As atletas passaram por um programa de treinamento proprioceptivo composto por seis exercícios que trabalharam propriocepção em diferentes graus de exigência durante quatro semanas e este teste foi aplicado no pré e pós-intervenção pelo programa de treinamento proprioceptivo das atletas. Resultados: As variáveis quantitativas foram descritas por média e desvio padrão, e o estudo da normalidade das variáveis foi descrito pelo teste de Shapiro-Wilk. As variáveis categóricas foram descritas por frequências absolutas e relativas. Para a comparação de médias de ambos os tornozelos pré e pós-intervenção foi utilizado o teste T pareado. O nível de significância adotado foi de $5 \%$ $(p \leq 0,05)$ e as análises foram realizadas no programa SPSS (Statistical Package for the Social Sciences) versão 18. Os resultados no teste SEBT das atletas pré e pós-intervenção proprioceptivo mostrou diferença significativa em seis direções para o tornozelo direito e cinco para o tornozelo esquerdo. Conclusão: Um programa de treinamento proprioceptivo parece ser eficaz para o incremento da estabilidade articular que é fundamental para atletas de voleibol expostas ao risco de entorses de tornozelo que geram instabilidades crônicas.

Palavras-chave: avaliação, estabilidade, propriocepção.

\begin{abstract}
Introduction: In sports where there is contact between athletes such as volleyball, sprain is the most common injury and the ankle is the joint most affected when compared with the others, causing major deficits such as joint instability. Proprioceptive training programs have been widely used to minimize these issues. Objective: To evaluate the effect of a proprioceptive training program on ankle joint stability using the Star Excursion Balance Test (SEBT) in volleyball athletes. Methods: A total of 11 volleyball players from a multi-sport club in Porto Alegre/RS/Brazil were evaluated through an assessment test for stability of the ankle joint, SEBT. The athletes underwent a proprioceptive training program consisting of six exercises that worked proprioception at different levels of difficulty for four weeks and this test was administered pre and post-intervention through the proprioceptive training program of the athletes. Results: Quantitative variables were described by mean and standard deviation, and the study of normality of the variables was described by the Shapiro-Wilk test. Categorical variables were described by absolute and relative frequencies. The paired $t$-test was used to compare the means of both ankles pre and post-intervention. The level of significance was $5 \%(p \leq 0.05)$ and analyses were performed using SPSS (Statistical Package for the Social Sciences) version 18. The results of the SEBT test pre and post proprioceptive intervention showed a significant difference in six directions for the right ankle and five for the left ankle. Conclusion: A proprioceptive training program seems to be effective for increasing joint stability which is essential for volleyball athletes at risk of ankle sprains that cause chronic instabilities.
\end{abstract}

Keywords: evaluation, stability, proprioception.

\section{RESUMEN}

Introducción: En los deportes en donde hay contacto entre los atletas, como en el vóleibol, la torsión por mecanismo de inversión es la lesión más frecuente y el tobillo es la articulación más acometida cuando comparada con las otras, produciendo déficits importantes como la inestabilidad articular. Los programas de entrenamiento propioceptivo han sido ampliamente utilizados para minimizar esas cuestiones. Objetivo: Evaluar el efecto de un 
entrenamiento propioceptivo en la estabilidad articular del tobillo por medio del test Star Excusion Balance Test (SEBT) en atletas de vóleibol. Métodos: Fueron evaluadas 11 atletas de vóleibol de un club polideportivo de la ciudad de Porto Alegre/RS/Brasil, por medio de un test de evaluación para estabilidad articular del tobillo, SEBT. Las atletas pasaron por un programa de entrenamiento propioceptivo compuesto por seis ejercicios que trabajaron propiocepción en diferentes grados de exigencia durante cuatro semanas y este test fue aplicado en la prey postintervención a través del programa de entrenamiento propioceptivo de las atletas. Resultados: Las variables cuantitativas fueron descritas por promedio y desvío estándar, yel estudio de la normalidad de las variables fue descrito a través del test de Shapiro-Wilk. Las variables categóricas fueron descritas por frecuencias absolutas y relativas. Para la comparación de promedios de ambos tobillos pre y postintervención fue utilizado el Test T apareado. El nivel de significancia adoptado fue de 5\% $(p \leq 0,05)$ y los análisis fueron realizados en el programa SPSS (Statistical Package for the Social Sciences) versión 18. Los resultados en el test SEBT de las atletas pre y post intervención propioceptivo mostró diferencia significativa en seis direcciones para el tobillo derecho y cinco para el tobillo izquierdo. Conclusión: Un programa de entrenamiento propioceptivo parece ser eficaz para el incremento de la estabilidad articular que es fundamental para atletas de vóleibol expuestas al riesgo de torsiones de tobillo que generan inestabilidades crónicas.

Palabras clave: evaluación, estabilidad, propiocepción.

\section{INTRODUÇÃO}

O voleibol está entre os esportes mais populares do mundo. Esta modalidade tem como gestos esportivos uma grande quantidade de saltos repetitivos, em diversos movimentos como o de defesa (bloqueio), os movimentos de armação de jogadas (levantamento) e os movimentos de ataque (saque e finalização das jogadas) ${ }^{1,2}$. Os saltos por sua vez são gestos que no retorno ao solo podem provocar uma inadequada aterrissagem e provocar lesões como as entorses ${ }^{3,4}$ Esses gestos tornam-se uma ameaça as articulações dos atletas devido a característica desse esporte ser de uma modalidade que muitas vezes permite o contato entre atletas ${ }^{1-3}$. É nesse contato que muitas vezes aparecem as lesões, sendo a entorse a lesão mais frequente e o tornozelo a articulação mais acometida quando comparada com as outras articulações ${ }^{1,4}$. As entorses correspondem a quase $80 \%$ dessas lesões e o mecanismo por inversão chega a $90 \%{ }^{5}$.

A entorse de tornozelo é claramente a lesão mais comum no voleibol e a maioria delas ocorre na zona de rede, durante o contato tanto com um companheiro de equipe ou um oponente, ou na aterrissagem após atacar ou bloquear ${ }^{1-3}$. Em uma entorse por inversão pode ocorrer a lesão do ligamento talofibular anterior, da região antero-lateral da cápsula articular e do ligamento calcâneo fibular, resultando na presença de dor aguda, redução da amplitude de movimento, déficit da função física ${ }^{6}$ e instabilidade, sendo esta última considerada a maior consequência desta lesão ${ }^{7,8}$. A instabilidade do tornozelo pode existir após uma ou mais entorses, sendo definida como a tendência do tornozelo a sofrer falseios, preferencialmente após as entorses do tipo grau II que muitas vezes se tornam instáveis cronicamente ${ }^{6-8}$.

Diversos estudos na literatura propõem protocolos de treinamento proprioceptivo que envolve a articulação do tornozelo com o objetivo de prevenir lesões e/ou diminuir os sintomas da instabilidade ${ }^{6,9-11}$. A propriocepção descreve a consciência de postura, de movimento e das mudanças de equilíbrio, englobando ainda o conhecimento da posição, do peso e da resistência dos objetos relacionado ao corpo ${ }^{7,12}$. É uma variação especializada da modalidade sensorial tátil e engloba as sensações de cinestesia, movimentação articular e sensação posicional ${ }^{12}$. Os receptores sensoriais se adaptam à estimulação constante. Esta adaptação dos receptores é considerada uma das mais importantes bases neurais para adaptação perceptual ${ }^{12}$, e isso explica os ganhos proporcionados pelo treinamento proprioceptivo ${ }^{6,9-11,13}$. Os déficits proprioceptivos são apontados pela literatura com propostas de testes avaliativos através da verificação do desempenho funcional de indivíduos com e sem insta- bilidade de tornozelo em diferentes situações ${ }^{14,15}$. Dentre as avaliações mais usadas atualmente, o Star Excursion Balance Test é um dos testes de equilíbrio considerado como ferramenta atual, de fácil manuseio, não instrumental e com uma relação custo-benefício satisfatória16,17.

O objetivo deste estudo foi avaliar o efeito de um treinamento proprioceptivo na estabilidade articular do tornozelo por meio do teste Star Excusion Balance Test (SEBT) em atletas de voleibol.

\section{MATERIAIS E MÉTODOS}

Foram avaliadas 11 atletas da modalidade voleibol de um clube poliesportivo da cidade de Porto Alegre, RS, Brasil. Todas as atletas eram do gênero feminino, com faixa etária entre 15 e 17 anos de idade (média $=16,09 \pm 0,70$ ) da categoria infanto-juvenil. Como critérios de inclusão, estas atletas tinham que ser praticantes da modalidade há no mínimo um ano, pertencer à mesma categoria com a mesma frequência de treinos (cinco vezes por semana), sem participação em qualquer programa de treinamento proprioceptivo específico para a articulação do tornozelo e participar do programa proprioceptivo proposto nesta pesquisa com no mínimo $80 \%$ de frequência, sem histórias de lesões nas articulações dos tornozelos.

Antes do início do procedimento de coleta dos dados todas as atletas e seus responsáveis assinaram o termo de consentimento livre e esclarecido aprovado pelo Comitê de Ética em Pesquisa da UFRGS juntamente com a proposta do projeto do estudo em 22/08/2013 sob o número de CAAE: 16159513.5.0000.5347 e número do parecer: 367.892, seguindo a Resolução no 196/96 do Conselho Nacional da Saúde.

Foi aplicado um questionário sob a forma de entrevista para composição da amostra de acordo com os critérios de inclusão e caracterização da amostra final para resultados. Cada atleta respondeu individualmente o questionário.

Após essa seleção, as atletas iniciavam a avaliação pré-intervenção no teste validado proposto para o estudo: o Start Excursion Balance Test (SEBT). Este teste consistia em uma série de mini agachamentos unilaterais realizados durante a tentativa de chegar o mais longe possível em uma determinada direção com a perna oposta. Foi confeccionada uma grande rosa-dos-ventos em um painel que ficava localizado no chão, com oito direções diferentes com ângulos de $45^{\circ}$ entre si, conforme estudo de Rasool e George ${ }^{18}$. A atleta era posicionada em apoio unipodal no centro dessa rosa-dos-ventos e a perna contra lateral realizava a extensão à frente, no ar, tentando como objetivo alcançar a maior distância possível em cada um dos 
sentidos. A mensuração foi realizada através da medição da distância a partir do centro da rosa-dos-ventos para o ponto mais distante alcançado pela ponta do pé em cada direção (figura 1). O teste foi aplicado em ambos os tornozelos. A proposta do teste consistia em quanto maior a distância atingida no momento da execução do teste, maior a demanda sobre os sistemas de equilíbrio e controle neuromuscular, apontando para uma maior estabilidade no tornozelo testado da atleta, permitindo assim, a mensuração de variáveis de equilíbrio com inferências a propriocepção ${ }^{18}$.

A partir da coleta do pré-teste, as atletas eram submetidas a um treinamento proprioceptivo específico para tornozelos, que foi baseado nos estudos de Hupperets et al. ${ }^{9}$, o qual consistiu na execução de seis exercícios com proposta de treino de propriocepção em diferentes graus de exigência, para ambos os tornozelos, e evolução semanal com o incremento do desequilíbrio através de uma superfície instável e incremento do senso de orientação através de uma venda nos olhos de acordo com a semana de treinamento. Essa proposta teve a duração de 12 encontros, realizados três vezes por semana, durante quatro semanas, e sua execução levava aproximadamente 20 minutos e foi inserido no treinamento físico das atletas, com a supervisão integral da pesquisadora.

Após as quatro semanas, todas as atletas foram submetidas à reavaliação de seus tornozelos através do teste SEBT na fase denominada pós-intervenção. Esta reavaliação era idêntica à avaliação realizada na pré-intervenção.

\section{Análise estatística}

As variáveis quantitativas foram descritas por média e desvio padrão, sendo que o estudo da normalidade das variáveis foi pelo teste de Shapiro-Wilk. As variáveis categóricas foram descritas por frequências absolutas e relativas. Para a comparação de médias de ambos os tornozelos pré e pós-intervenção foi utilizado o Teste T pareado. O nível de significância adotado foi de $5 \%(p \leq 0,05)$ e as análises foram realizadas no programa SPSS (Statistical Package for the Social Sciences) versão 18.

\section{RESULTADOS}

A amostra foi composta por 11 atletas do gênero feminino com média de idade de 16,09 anos $\pm 0,70$. As características antropométricas mostraram uma média de estatura de $1,75 \mathrm{~m} \pm 0,07$ e peso de $68,45 \mathrm{Kg} \pm 0,09$ destas atletas. Com relação ao tempo de prática, a média foi de 5,82 $\pm 1,17$ anos.
Após o treinamento proprioceptivo proposto, o desempenho dos tornozelos das atletas pré e pós-intervenção pelo teste SEBT foi comparado, e os resultados mostraram significância estatística em diversas direções, tanto para o tornozelo direito, quanto para o tornozelo esquerdo.

Na comparação pré e pós-intervenção para o tornozelo direito, os resultados mostraram diferença estatística melhorando a estabilidade em pelo menos seis direções (anterior, lateral, postero-lateral, posterior, medial e antero-medial) das oito propostas no SEBT teste (tabela 1).

Na comparação pré e pós-intervenção para o tornozelo esquerdo, os resultados também mostraram diferença estatística melhorando a estabilidade em pelo menos cinco direções (anterior, lateral, postero-lateral, postero-medial e medial) das oito propostas no SEBT teste (tabela 2).

Tabela 1. Resultados do SEBT pré e pós-intervenção para o tornozelo direito.

\begin{tabular}{|c|c|c|}
\hline Variáveis & Média & Desvio padrão \\
\hline Tornozelo Direito & & \\
\hline Anterior MED pré & 85,93 & 6,15 \\
\hline Anterior MED pós & 93,05 & 9,07 \\
\hline $\mathrm{P}$ & \multicolumn{2}{|c|}{$0,015^{*}$} \\
\hline Anterolateral MED pré & 75,83 & 5,59 \\
\hline Anterolateral MED pós & 79,29 & 7,56 \\
\hline$P$ & \multicolumn{2}{|c|}{0,180} \\
\hline Lateral MED pré & 58,70 & 8,64 \\
\hline Lateral MED pós & 65,30 & 7,62 \\
\hline $\mathrm{P}$ & \multicolumn{2}{|c|}{$0,027^{*}$} \\
\hline Posterolateral MED pré & 90,94 & 7,33 \\
\hline Posterolateral MED pós & 97,67 & 4,96 \\
\hline $\mathrm{P}$ & \multicolumn{2}{|c|}{$0,004^{*}$} \\
\hline Posterior MED pré & 98,81 & 7,57 \\
\hline Posterior MED pós & 104,31 & 5,69 \\
\hline$P$ & \multicolumn{2}{|c|}{$0,008^{*}$} \\
\hline Posteromedial MED pré & 96,31 & 6,67 \\
\hline Posteromedial MED pós & 101,30 & 7,12 \\
\hline $\mathrm{P}$ & \multicolumn{2}{|c|}{0,053} \\
\hline Medial MED pré & 86,90 & 9,60 \\
\hline Medial MED pós & 94,74 & 8,38 \\
\hline $\mathrm{P}$ & \multicolumn{2}{|c|}{$0,011^{*}$} \\
\hline Anteromedial MED pré & 90,90 & 6,83 \\
\hline Anteromedial MED pós & 95,56 & 8,28 \\
\hline$P$ & \multicolumn{2}{|c|}{$0,043^{*}$} \\
\hline
\end{tabular}

\section{${ }^{*} \mathrm{p} \leq 0,05$.}

\section{DIREÇÕES UTILIZANDO O TORNOZELO ESQUERDO COMO APOIO}

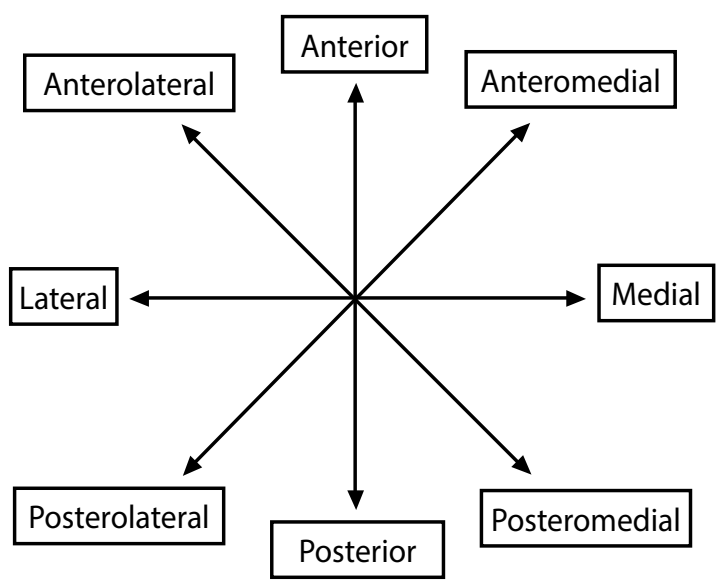

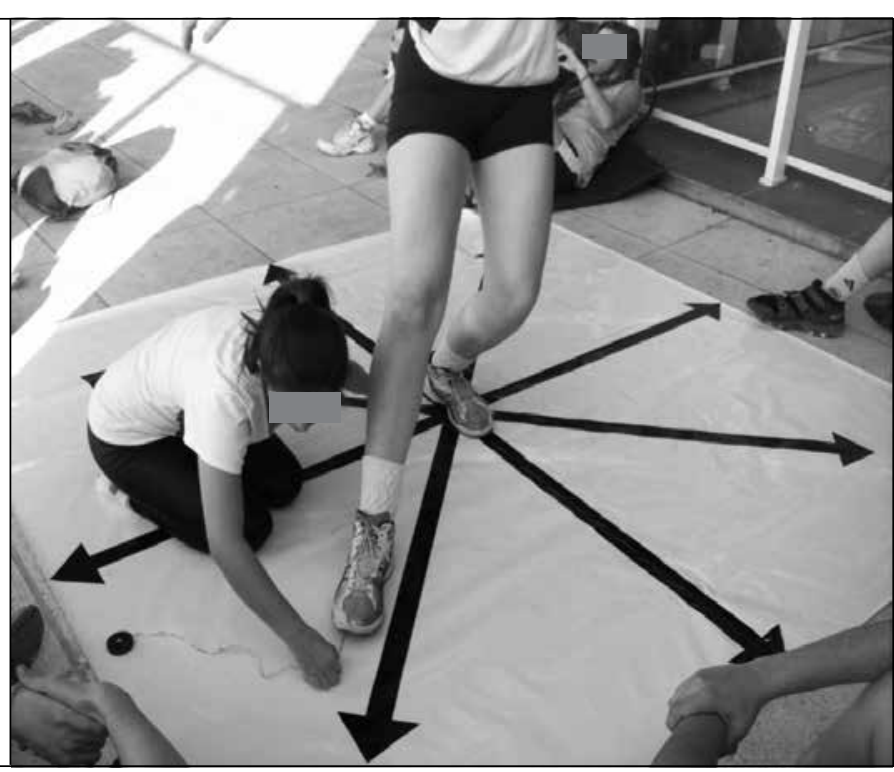

Figura 1. Diagrama para medida da distância do pé no Star Excursion Balance Test (SEBT). 
Tabela 2. Resultados do SEBT pré e pós-intervenção para o tornozelo esquerdo.

\begin{tabular}{|c|c|c|}
\hline Variáveis & Média & Desvio padrão \\
\hline \multicolumn{3}{|l|}{ Tornozelo Esquerdo } \\
\hline Anterior MED pré & 90,24 & 5,23 \\
\hline Anterior MED pós & 93,16 & 7,34 \\
\hline $\mathrm{P}$ & \multicolumn{2}{|c|}{$0,050^{*}$} \\
\hline Anterolateral MED & 92,50 & 3,74 \\
\hline Anterolateral MED pós & 94,87 & 7,51 \\
\hline$P$ & \multicolumn{2}{|c|}{0,129} \\
\hline Lateral MED pré & 57,77 & 5,64 \\
\hline Lateral MED pós & 62,55 & 6,06 \\
\hline$P$ & \multicolumn{2}{|c|}{$0,022^{*}$} \\
\hline Posterolateral MED pré & 92,36 & 8,16 \\
\hline PosterolateralMED pós & 97,37 & 7,16 \\
\hline$P$ & \multicolumn{2}{|c|}{$0,013^{*}$} \\
\hline Posterior MED pré & 101,90 & 7,80 \\
\hline Posterior MED pós & 103,21 & 6,79 \\
\hline $\mathrm{P}$ & \multicolumn{2}{|c|}{0,469} \\
\hline Posteromedial MED pré & 98,46 & 6,08 \\
\hline Posteromedial MED pós & 103,55 & 7,24 \\
\hline$P$ & \multicolumn{2}{|c|}{$0,014^{*}$} \\
\hline Medial MED pré & 91,73 & 7,90 \\
\hline Medial MED pós & 98,28 & 8,29 \\
\hline $\mathrm{P}$ & \multicolumn{2}{|c|}{$0,003^{*}$} \\
\hline Anteromedial MED pré & 92,50 & 6,67 \\
\hline Anteromedial MED pós & 94,87 & 8,17 \\
\hline$P$ & \multicolumn{2}{|c|}{0,210} \\
\hline
\end{tabular}

${ }^{*} p \leq 0,05$.

\section{DISCUSSÃO}

Este estudo buscou verificar os efeitos de um programa de treinamento proprioceptivo com jovens atletas de voleibol, em que o treinamento proposto mostrou-se eficaz no incremento da propriocepção das atletas avaliadas através do SEBT, teste que demonstra a estabilidade articular do tornozelo relacionado com as lesões músculo-esqueléticas desta articulação ${ }^{14}$. Sendo assim, após o treinamento proprioceptivo proposto, estas atletas apresentaram melhora da estabilidade articular do tornozelo contribuindo para menor suscetibilidade a lesões nessa articulação, principalmente a entorse de tornozelo, lesão de maior ocorrência na prática do voleibol.

Existem outras metodologias que, além do programa proprioceptivo, também se propõem a avaliar a estabilidade articular do tornozelo como o tempo de reação muscular, através da eletromiografia de superfície, e a estabilidade postural, através da plataforma de força, a qual muito se assemelha a proposta avaliativa do SEBT teste. Nesse sentido Isakov e Mizrahi ${ }^{19}$ utilizaram uma plataforma de força para mensurar as forças de reação entre as direções antero-posterior e médio-lateral durante o apoio unipodal em tornozelos com e sem entorse, e nas situações de olhos abertos e fechados. Os resultados apresentados para os controles nas direções antero-posterior e médio-lateral foram os mesmos entre os sujeitos com entorse crônica e sujeitos sem lesão, enquanto estes estavam de olhos abertos ou fechados.

Todas essas avaliações propostas pela literatura são de suma importância para entendermos a propriocepção que é um tema fundamental quando se fala em estabilidade articular. A propriocepção pode ser definida como uma variação do tato somada às sensações de posição, movimento articular (cinestesia), por receptores cutâneos de planta do pé, cápsulas articulares e ligamentos ${ }^{7,20}$.

Quando ocorre uma lesão como a entorse de tornozelo, os proprioceptores ou mecanorreceptores ficam danificados, prejudicando assim a propriocepção ${ }^{21,22}$. Para Freeman et al.7, o mecanismo básico da instabilidade do tornozelo desenvolvido pela entorse é devido à lesão dos proprioceptores na cápsula articular e nos ligamentos. Essa instabilidade causa descoordenação motora, com interferência dos reflexos

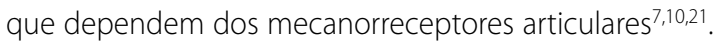

Especificamente para a proposta de exercícios proprioceptivos como incremento para aumentar a estabilidade articular do tornozelo, a presente pesquisa corrobora estudos que demonstraram que esses exercícios têm uma grande ação profilática e de reabilitação em lesões musculoesqueléticas, pois exigem da modalidade sensorial uma forma mais competente para obtenção de informações referentes à sensação de movimento e posição articular22.

O estudo de Sheth et al. ${ }^{23}$ propõe um treinamento proprioceptivo em 10 sujeitos com tornozelos direito dominantes, sem lesão em uma plataforma de instabilidade para avaliar o tempo de reação muscular. Os sujeitos deste grupo experimental passaram por sessões de treinamento na plataforma durante oito semanas. Na fase do pós-treino, os resultados mostraram que esse treinamento desenvolveu um padrão de contração muscular que favorecia a correção da inversão excessiva da articulação do tornozelo em situações de entorse, contribuindo para uma melhor estabilidade articular.

Dessa forma, estudos que propõem protocolos de treinamento proprioceptivo para avaliação da estabilidade articular têm sido apontados como referência padrão para a comparação com a instabilidade. Nesse sentido, esta pesquisa utilizou um protocolo baseado no estudo de Hupperets et al. ${ }^{9}$ que propõem seis exercícios que envolveram equilíbrio estático e dinâmico para avaliação dos déficits da performance funcional da articulação do tornozelo pelo teste SEBT.

O incremento destes treinamentos também são importantes para bons resultados, como mostrou o estudo de Oliveira et al. ${ }^{24}$ que durante a realização de exercícios proprioceptivos de olhos vendados apresentou uma ativação muscular maior dos estabilizadores dinâmicos, quando comparado com a realização de exercício de olhos abertos, mostrando assim a influência do sistema visual na propriocepção corporal. Além disso, o Colégio Americano de Medicina do Esporte recomenda que um aumento no grau de dificuldade dos exercícios de equilíbrio pode ser realizado pela progressiva redução da base de apoio, com movimentos dinâmicos que perturbam o centro de gravidade, destacando músculos posturais ou reduzindo as entradas sensoriais ${ }^{25}$. Ainda segundo Borreani ${ }^{25}$, exercícios que utilizam a postura unipodal demandam maior ativação dos músculos estabilizadores do tornozelo.

Os estudos de Hupperets et al. ${ }^{9}$ e Verhagen et al. ${ }^{6}$ utilizaram protocolos semelhantes ao do presente estudo para o treinamento proprioceptivo com atletas e obtiveram resultados satisfatórios em relação a prevenção da entorse de tornozelo. Hupperets et al. ${ }^{9}$ propuseram uma intervenção de oito semanas e conseguiram atingir uma redução de $35 \%$ no risco de recorrência de entorse. Da mesma forma, Verhagen et al. 6 utilizaram um protocolo que abrangeu atletas de voleibol de 116 times e obtiveram resultados significativos em relação a diminuição da entorse de tornozelo no grupo intervenção. Estes estudos, assim como o presente estudo, apontam para a eficácia do treinamento proprioceptivo na estabilidade articular em atletas.

Outro estudo de O'Driscoll et al..$^{10}$ que propõem um treinamento progressivo envolvendo a propriocepção, com frequência de três vezes por semana e duração de seis semanas, utilizaram como forma de avaliação pré e pós treinamento quatro diferentes avaliações entre elas o SEBT teste. Foram selecionadas para a análise apenas três direções: anterior, postero-medial e postero-lateral e foi encontrado resultado significativo quando comparados pré e pós-intervenção. O presente estudo também encontrou resultados estatisticamente significativos 
para essas direções em relação ao tornozelo esquerdo e para as direções anterior e posterolateral na análise para o tornozelo direito.

O estudo de Hertel et al. ${ }^{17}$ avaliou as direções que seriam mais relevantes em uma avaliação clinica para testar os déficits funcionais relacionadas com a instabilidade de tornozelo. Como resultados, obtiveram que as direções anteromedial, medial e posteromedial foram as mais relevantes. No presente estudo, as análises dessas direções mostraram resultados significativos. Em relação ao tornozelo direito apresentaram um efeito com relevância em duas dessas três direções e em relação ao tornozelo esquerdo, também apresentaram um efeito com relevância na direção medial e em outra dessas três direções apontadas no estudo.

O treinamento proprioceptivo também obteve resultado benéfico em atletas de outras modalidades e em sujeitos saudáveis e destreinados para o esporte. No estudo de Baldaço et al., ${ }^{11}$ foi proposto um treinamento proprioceptivo para atletas de futsal feminino com frequência de treino de três vezes por semana durante um mês, e como forma de avaliação pré e pós-treinamento foi utilizada uma plataforma de força. Os resultados apresentaram-se estatisticamente significativos, sugerindo que o protocolo de propriocepção demonstrou maior controle de equilíbrio postural nas condições e na amostra avaliada.

Também encontramos na literatura propostas de treinamento para mulheres não atletas e saudáveis. $O$ estudo de Braga et al. ${ }^{13}$ propuseram um treinamento proprioceptivo para dois grupos de mulheres, as quais também foram avaliadas através do SEBT. Um grupo realizou o treinamento através do dispositivo Nintendo Wii e outro os sujeitos desenvolveram exercícios em um disco proprioceptivo. Ambos apresentaram melhora significativa no desempenho do SEBT antes e depois do treinamento.

Em vista dos argumentos apresentados, a literatura aponta que esses programas de treinamento neuromuscular ou treinamentos proprioceptivos são muito importantes e bem sucedidos tanto na prevenção de casos recorrentes de entorse de tornozelo, quanto do ponto de vista de eficácia de tratamento, melhorando a estabilidade articular e também de perspectiva de $\operatorname{custos}^{6,9,26}$. Além disso, sugere-se o uso de plataformas de equilíbrio que produzem maior atividade muscular do tornozelo em comparação com superfícies planas ou trampolins ${ }^{27}$.

Porém, como cita Verhagen, ${ }^{26}$ mesmo com conhecimento dos mecanismos de estabilidade, ainda falta uma visão fundamental para o uso de mecanismos preventivos, e essa citação vem ao encontro do que ocorreu no presente estudo, onde é importante destacar que as atletas já praticavam treinamento de propriocepção preventivo para os membros inferiores, não específicos para os tornozelos, e por esse motivo, talvez essa prevenção tenha contribuído para o incremento da estabilidade articular encontrada nos resultados desta pesquisa.

\section{CONCLUSÃO}

Baseado nos resultados obtidos pode-se concluir que o treinamento proprioceptivo em relação à articulação do tornozelo foi eficaz mesmo em uma amostra pequena onde foram encontradas diferenças entre as médias pré e pós-intervenção em diferentes direções, mostrando o incremento da estabilidade adquirida por esta articulação. Porém, não se pode afirmar que somente estas quatro semanas de treinamento proprioceptivo específico para os tornozelos foram responsáveis por melhorar a estabilidade articular, pois as atletas do estudo já praticavam treinamento proprioceptivo prévio. Pode-se apenas apontar para uma tendência de melhora desta estabilidade.

Para esta pesquisa, assim como na literatura, a variação metodológica, o tamanho amostral, os cálculos estatísticos e diferentes populações são fatores que poderão interferir em muitos resultados.

Todos os autores declararam não haver qualquer potencial conflito de interesses referente a este artigo.

\section{REFERÊNCIAS}

1. Pacheco AM, Vaz M, Pacheco I. Avaliação do tempo de resposta eletromiográfica em atletas de voleibol e não atletas que sofreram entorse de tornozelo. Rev Bras Med Esporte. 2005;11:325-30.

2. Meurer MC; Pacheco I; Silva MF; Pacheco AM. Análise da influência da bandagem funcional de tornozelo no tempo de reação do fibular longo em sujeitos saudáveis. Rev Bras Med Esporte. 2010;16(3):196-200

3. Verhagen EALM, Van Der Beek AJ, Bouter LM, Bahr RM, Van Mechelen W. A one season prospective cohort study of volleyball injuries. Br J Sports Med. 2004;38:477-81.

4. Cardoso JR, et al. Influência da utilização da órtese de tornozelo durante atividades do voleibol: avaliação eletromiográfica. Rev Bras Med Esporte. 2005;11(5):276-80.

5. Junge A, Langevoort G, Pipe A, Peytavin A, Wong F, Mountjoy M, Beltrami G, Terrell R, Holzgraefe M, Charles R, Dvorak J. Injuries in team sport tournaments during the 2004 Olympic Games. Am J Sports Med. 2006; 34(4):565-76.

6. Verhagen EALM, Van der Beek AJ, Twisk JWR, Bouter L, Bahr R, Van Mechelen W. The effect of a proprioceptive balance board training program for the prevention of ankle sprains: a prospective controlled trial. Am J Sports Med 2004;32:1385-93.

7. Freeman MA. Instability of the foot after injuries to the lateral ligament of the ankle. J Bone Joint Surg Br. 1965;47:669-77.

8. Prado MP; Fernandes TD, Camanho GL, Mendes AAM, Amodio DT. Mechanical instability after acute ankle ligament injury: randomized prospective comparison of two forms of conservative treatment. Rev Bras Ortop. 2013;48(4):307-16

9. Hupperets MDW, Verhagen EALM, Van Mechelen W. Effect of unsupervised home based proprioceptive training on recurrences of ankle sprain: randomised controlled trial. BMJ. 2009;339:b2684.

10. O'Driscoll J, Kerin F, Delahunt E. Effect of a 6-week dynamic neuromuscular training programme on ankle joint function: A Case report. Sports Medicine, Arthroscopy, Rehabilitation, Therapy \& Technology. 2011;3:13;1-7.

11. Baldaço FO, Cado VP, Souza J, Mota CB, Lemos JC. Análise do treinamento proprioceptivo no equilíbrio de atletas de futsal feminino. Fisioter Mov. 2010; 23:183-92.

12. Westlake KP, Wu Y, Culham, EG. Sensory-Specific Balance Training in Older Adults: Effect on Position, Movement, and Velocity Sense at the Ankle. Phys Ther. 2007;87(5):560-8.

13. Braga MM, Nunes GS, Schutz GR, Menezes FS. Treinamento sensório-motor com Nintendo Wii ${ }^{\circledR}$ e disco proprioceptivo: efeitos sobre o equilíbrio de mulheres jovens saudáveis. R. Bras. Ci. e Mov. 2012;20:37-45.
14. Suda EY, Souza RB. Análise da performance funcional em individuos com instabilidade do tornozelo: Uma Revisão Sistemática da Literatura. Rev Bras Med Esporte. 2009;15:233-37.

15. Olmsted LC, Garcia CR, Hertel J, Shultz SJ. Efficacy of the star excursion balance tests in detecting reach deficits in subjects with chronic ankle instability. J Athl Train. 2002;37:501-6.

16. Meneghini T, Rempel C, Barnes CD, Périco E, Duarte F. Avaliação da ativação proprioceptiva em atletas amadoras de voleibol. Com Scientia e Saúde, 2009;8:47-55.

17. Hertel J, Braham RA, Hale SA, Olmsted-Kramer LC. Simplifying the Star Excursion Balance Test: Analyses of Subject Wich and Without Chronic Ankle Instability. J Ortop Sports Phys Ther. 2006; 36(3):131-7.

18. Rasool J, George K. The impact of single-leg dynamic balance training on dynamic stability. Phys Ther in Sport, 2007:8(4):177-84.

19. Isakov E, Mizrahi J. Is balance impaired by recurrent sprained ankle? Br. J. Sports Med. 1997;31(3):65-7.

20. Lokhande MV, Shetye J, Mehta A, Deo MV. Assessment of knee joint proprioception in weight bearing and in non-weight bearing positions in normal subjects. JKIMSU. 2013;2(2):94-101.

21. Souza RZ, Bastos FN, Vanderlei LCM, Júnior JN, Pastre CM. Atualização sobre modelos de avaliação e treinamento proprioceptivo para articulação do tornozelo. Terapia Manual. 2010; 8(40):179-82.

22. Westlake KP, Wu Y, Culham EG. Sensory-Specific Balance Training in Older Adults: Effect on Position, Movement, and Velocity Sense at the Ankle. Phys Ther. 2007;87(5):560-8.

23. Sheth P, Yu B, Laskowski ER, An KN. Ankle disk training influences reaction times of selected muscles in a simulated ankle sprain. Am J Sports Med. 1997;25(4):538-43.

24. Oliveira DCS, Santos PAM, Rezende L, Silva MR, Lizardo FB, Sousa GC, Santos LA, Guimarães EA et al. Análise eletromiográficaa de músculos do membro inferior em exercícios proprioceptivos realizados com olhos abertos e fechados. Rev Bras Med Esporte. 2012;18:262-6.

25. Borreani S, Calatayud J, Martin J, Colado JC, Tella V, Behm D. Exercise intensity progression for exercises performed on unstable and stable platforms based on ankle muscle activation. Gait \& Posture 2014;39(1):404-9.

26. Verhagen EALM. How fundamental knowledge aids implementation: ankle sprains as an example. Acta Med Port. 2013;26(2):171-4.

27. Cimadoro G, Paizis C, Alberti G, Babault N. Effects of different unstable supports on EMG activity and balance. Neuroscience Letters 2013;548:228-32. 